\title{
Lingüistica clínica en México, ¿por qué y para qué?
}

\author{
Clinical linguistics in Mexico, \\ why and what for?
}

Josaphat Enrique

Guillén Escamilla Escuela Nacional de Antropología

e Historia, Licenciatura en Lingüística josaphat_guillen@enah.edu.mx 


\section{Resumen}

Esta nota presenta un panorama histórico del desarrollo de la lingüística clínica (LC) en México. En un primer momento, se establece la distinción entre psicolingüística, neurolingüística y LC, ya que dichos términos tienden a utilizarse de manera indistinta. Posteriormente, se realiza un recorrido histórico sobre las investigaciones que sentaron las bases de la LC, partiendo de la década de los 70 del siglo pasado. También, se subrayan las principales dificultades a las que se ha enfrentado esta disciplina, mismas que han afectado su desarrollo. Finalmente, se destaca la necesidad de consolidar esta línea de investigación — como parte de la lingüística aplicada - sobre todo, por su posible contribución a la optimización de la práctica clínica de terapeutas del lenguaje $y$, en consecuencia, al mejoramiento de la calidad de vida de pacientes con alguna alteración del lenguaje, así como la de sus familiares.

Palabras clave: lingüística aplicada; afasia; patologías del lenguaje; alteraciones del lenguaje; neuropsicología del lenguaje

\section{Abstract}

This article introduces a historic perspective about the development of Clinical Linguistics (CL) in Mexico. First, it establishes a distinction between psycholinguistics, neurolinguistics, and CL, because these three subfields tend to be used indistinctly. It continues with a historic review about researches and researchers that contributed to set the basis of $\mathrm{CL}$, starting in the 1970s. Also, it highlights the main difficulties facing CL that have restricted its development. Finally, it stresses the need to consolidate this line of research as part of Applied Linguistics, especially because CL can contribute to optimize the clinical practice of speech therapists and, consequently, improve the quality of life of patients with speech disorders and their relatives.

Keywords: Applied Linguistics; aphasia; speech pathology; speech disorders; neuropsychology of language 


\section{Introducción}

La lingüística clínica (LC) es una rama de la lingüística aplicada, relativamente reciente. En el mundo anglosajón apareció durante los primeros años de la década de 1970, pero no fue sino hasta inicios de los años 80 que se constituyó como tal (Crystal, 1981); mientras que en España y Sudamérica —en Chile, específicamente- lo hizo hacia finales de los 90 (Fernández, 1998; Pavez, 1998). Desde entonces, los estudios del área se han centrado en dos aspectos: i) sentar las bases teóricas de la LC y destacar la importancia de la aplicación de la lingüística en el ámbito clínico, y ii) el análisis de trastornos lingüísticos desde esta nueva perspectiva. A pesar de esta tradición en el ámbito hispánico, en México la LC sigue siendo un campo poco explorado. Esto no implica una falta de interés en el estudio de las alteraciones del lenguaje; más bien, deja al descubierto que los profesionales en psicología, neuropsicología, terapia del lenguaje, etcétera, han sido los principales interesados, aun cuando puedan carecer de los conocimientos específicos para llevar a cabo tal empresa o persigan fines muy distintos a los de la LC.

Esta situación se agrava cuando notamos que los términos psicolingüística, neurolingüística y lingüística clínica tienden a emplearse de manera indistinta, como si el objeto de estudio, la metodología, los materiales y los intereses de investigación fueran los mismos. La consecuencia obvia de esta imprecisión es que no solo no se tiene claro qué es la LC, sino que tampoco se sabe con certeza qué es neurolingüística y qué es psicolingüística. Ante este escenario, lo que intentamos hacer en este trabajo es cumplir con cuatro objetivos principales: i) trazar los lindes entre neurolingüística, psicolingüística y LC, ii) presentar un panorama general de la historia, alcances y objetivos de la LC, iii) describir cuál es la situación actual de los estudios sobre las alteraciones del lenguaje en México y iv) proponer algunos caminos para el desarrollo de la LC, atendiendo principalmente a los retos que supone una disciplina con sus características y bajo las condiciones particulares de nuestro país. 


\section{La lingüistica y la relación cerebro-mente-lenguaje: psicolingüistica y neurolingüistica}

Delimitar los alcances y objetivos de estas dos ramas no es tarea fácil, sobre todo porque a priori comparten el mismo objetivo: el estudio de la relación cerebro-mente-lenguaje. Consideramos que una buena forma de iniciar la exposición es cronológicamente, dado que existe un consenso sobre el nacimiento de cada una de ellas, así como los hechos que contribuyeron a su consolidación como disciplinas independientes. También conviene señalar que nuestro interés no es realizar un recorrido histórico exhaustivo, por lo que nos limitaremos a abreviar los acontecimientos más importantes del siglo xx, aquellos que permitieron el establecimiento de cada una de estas ramas.

\subsection{La psicolingüística}

Los estudios realizados durante el siglo XIX por Paul Broca (1865) y Carl Wernicke (1874) pueden ser considerados como los antecedentes directos de lo que a mediados del siglo xx se conocería como psicolingüística (Garnham, Garrod \& Sanford, 2006). El nacimiento de esta disciplina — tal y como hoy la conocemostuvo lugar en 1951, durante un encuentro del Social Science Research Council en la Universidad de Cornell, donde se fundó un comité sobre lingüística y psicología que tuvo como presidente a Charles Osgood (Fernández \& Smith, 2011). Posteriormente, en 1954, aparecería la obra fundacional: Psycholinguistics: A survey of theory and research problems, de Osgood y Sebeok (Garnham, Garrod \& Sanford, 2006).

En ese mismo año, las novedosas ideas de Chomsky - sobre competencia lingüística, innatismo y, en general, la naturaleza mentalista de su teoría- impulsaron enormemente el interés de los investigadores por la psicolingüística y comenzaron a desarrollarse trabajos en adquisición del lenguaje, procesamiento de la información y enseñanza de L1 y L2 bajo esta nueva perspectiva. 
En ese punto, la psicolingüística estaba interesada en descubrir "how people acquire language, how people use language to speak and understand one another, and how language is represented and processed in the brain"1 (Fernández \& Smith, 2011: 1).

Actualmente, existen varias definiciones operativas sobre el objetivo, los métodos y los fines de la psicolingüística. Algunas de ellas son escuetas y no reflejan cabalmente los alcances de la disciplina; otras más tienden a ser muy vagas y dificultan establecer una distinción clara con áreas cercanas, como la neurolingüística. Bajo estas circunstancias, resulta necesario plantear una definición que sirva como punto de partida común, con este fin retomamos la propuesta de Field:

Psycholinguistics is the study of how the mind equips human beings to handle language. Its central concern is with the $\operatorname{cog}$ nitive processes that underlie the storage, use and acquisition of language, and their correlates in observable neural activity in the brain. They include how language evolved, whether and why it is restricted to the human race, what the precise relationship is between language and thought and whether language shares functions with general cognition or operates independently of it $^{2}$ (2011: 472, énfasis añadido).

De acuerdo con esta definición, la psicolingüística exige construir un marco teórico interdisciplinar, donde confluyen la psicología

1 “[C]ómo las personas adquieren el lenguaje, cómo lo usan para hablar y comprender al otro, y cómo se representa y procesa en el cerebro" (Las traducciones de las citas son del autor).

2 "La psicolingüística es el estudio de cómo la mente dota a los seres humanos para manejar el lenguaje. Su preocupación central son los procesos cognitivos que subyacen al almacenamiento, uso y adquisición del lenguaje, y sus correlatos en la actividad neural observable en el cerebro. Incluyen cómo evolucionó el lenguaje, si está restringido a la raza humana y por qué, cuál es la relación precisa entre lenguaje y pensamiento y si el lenguaje comparte funciones con la cognición general u opera independientemente de ella." 
cognitiva, modelos cognitivos, la lingüística teórica, la fonética, la neurolingüística, la lingüística clínica, la pragmática y el análisis de discurso (Fernández \& Smith, 2011; Field, 2011). Todas estas áreas, en conjunto, contribuyen a explicar: i) los procesos cognitivos ${ }^{3}$ que están detrás de la producción, percepción y comprensión del lenguaje (Weismer, 2006); ii) cómo se almacena y emplea el vocabulario (Ahlsén, 2006), iii) el desarrollo filogenético y ontogenético del lenguaje (Field, 2011) y iv) cómo puede perderse la capacidad linguiística (Field, 2004; Ahlsén, 2006).

Así, una parte importante de la empresa psicolingüística reside en "la elaboración de modelos 4 [de los procesos cognitivos], sirviéndose de datos y evidencias obtenidas a través de experimentos y pruebas" (Fernández, 1998: 407), esto es, gracias a las técnicas de imaginería cerebral, propias de la neurolingüística, se ha podido confirmar y refinar las hipótesis sobre cómo trabaja la mente humana en conjunto con el lenguaje (Ahlsén, 2006; Field, 2011). De tal forma, queda ahora revelada la relación que existe entre ambas disciplinas. Por un lado, la psicolingüística retoma los resultados de la neurolingüística y los incorpora a los propios; además, se nutre de las técnicas que se emplean en esta última, particularmente la imaginería cerebral y el registro de la actividad durante tareas específicas, tales como repetición de palabras, comprensión de oraciones, lectura, entre otras. Y, por otro lado, la psicolingüística trata de explicar, además, las alteraciones del lenguaje a partir de los procesos que se encuentran afectados — como atención y memoria- planteando conclusiones empíricas, incluso sin apelar a las bases orgánicas cerebrales (Fernández, 1998). Así, en el caso de las afasias, ${ }^{5}$ "no sólo importan por sus características clínicas

3 Se entiende por proceso cognitivo una serie de operaciones mentales que nos permiten alcanzar un tipo de producto mental superior. Entre ellos, destacan: atención, memoria, percepción, etcétera (Runco, 2001).

4 Un ejemplo muy representativo es el modelo de producción del lenguaje de Levelt (1989), que tiene gran influencia en la psicolingüística actual.

5 Para Goodglass, Kaplan y Barresi (2001: 5), la afasia es "the disturbance of any or all of the skills, associations and habits of spoken and written language pro- 
sino por el contraste entre la actividad linguiística de los afásicos y la actividad lingüística en situaciones normales" (Fernández, 1998: 407).

A manera de sumario, podemos decir entonces que el interés principal de la psicolingüística está centrado en describir los procesos cognitivos que intervienen en la adquisición, desarrollo, enseñanza, comprensión y expresión del lenguaje, aun cuando pueda prescindir del estudio de las bases orgánicas subyacentes. Por otra parte, también estudia los procesos de pérdida del lenguaje, ya que puede contribuir a la comprensión del funcionamiento del lenguaje en condiciones naturales, principal objetivo de la psicolingüística.

\subsection{La neurolingüística}

Los orígenes del término neurolingüística se pueden rastrear a mediados de la década de los 70 del siglo pasado. Ahlsén (2011) sostiene que Harry Whitaker acuñó el término para presentar una serie de publicaciones, Studies in Neurolinguistics (1976-1979), aunque Ingram (2007: 3) plantea que —desde 1971 — Whitaker lo utilizaba para referirse a ciertos estudios: "language processing in the human brain and, more specifically, what happens to spoken language when certain areas of the brain are damaged". ${ }^{6}$ En este punto cabe señalar que, por los mismos años, en la Unión Soviética, Alexander Luria había publicado Basic problems of Neurolinguistics (Osnovnye problemy neirolingvistiki) (1976 [1975]), como continuación de algunos trabajos previos de la década de los 60 . No obstante, debido a las tensiones políticas de esos años, el tra-

duced by injury to certain brain areas that are specialized for these functions" ["la alteración de cualquiera o todas las habilidades, asociaciones y hábitos del lenguaje hablado y escrito producida por lesiones en ciertas áreas del cerebro que están especializadas para estas funciones"].

6 "[P]rocesamiento del lenguaje en el cerebro humano y, más específicamente, qué le pasa al lenguaje hablado cuando ciertas áreas del cerebro están dañadas." 
bajo de Luria tardó un poco más de tiempo en ser conocido en el mundo occidental.

Como en el caso de la psicolingüística, comenzamos presentando una definición de neurolingüística que nos sirva como punto de partida común:

Neurolinguistics studies the relation of language and communication to different aspects of brain function, in other words it tries to explore how the brain understands and produces language and communication. This involves attempting to combine neurological/neurophysiological theory (how the brain is structured and how it functions) with linguistic theory (how language is structured and how it functions) ${ }^{7}$ (Ahlsén, 2006: 3, énfasis añadido).

Así, la neurolingüística es un campo interdisciplinar relacionado tradicionalmente con la neurología y la fisiología (Whitaker \& Whitaker, 1976; Fernández, 1998), pero que hoy en día involucra, además, a las neurociencias, la psicología, la lingüística, la patología del habla y la biología (Ahlsén, 2011: 460). De tal suerte, sus métodos de investigación incluyen: imaginería cerebral, estimulación de procesos cerebrales y videograbación de interacciones verbales (Ahlsén, 2011).

La neurolingüística está interesada fundamentalmente en las bases orgánicas o estructuras cerebrales involucradas en la producción y comprensión del lenguaje, por lo que resulta natural que su estudio se haya centrado principalmente en los desórdenes del lenguaje provocados por daño cerebral (Garayzábal \& Otero, 2005;

7 "La neurolingüística estudia la relación del lenguaje y la comunicación con respecto a diferentes aspectos de la función cerebral, en otras palabras, intenta explorar cómo el cerebro comprende y produce el lenguaje y la comunicación. Esto supone una tentativa de combinar la teoría neurológica/neurofisiológica (cómo se estructura el cerebro y cómo funciona) con la teoría lingüística (cómo se estructura el lenguaje y cómo funciona)." 
Ingram, 2007; Stavrakaki, 2012). De tal forma, estudia a los hablantes - a su cerebro propiamente- más que al lenguaje, aunque analiza sus alteraciones para descubrir cuáles son los mecanismos neurológicos subyacentes de dichos déficits: "[s]uch comprehension is possible only if the language disorder is viewed in relation to the other central deficits which the patient may have"s (Lebrun, 1983: 243). En adición, en los últimos años, ha surgido un interés creciente por el estudio de la relación filogenética y ontogenética de cerebro, lenguaje y comunicación (Ahlsén, 2011).

De acuerdo con sus fines, algunos autores consideran que la neurolinguiística tiene un carácter más teórico (Fernández, 1998), aunque otros sostienen que tiene una orientación más aplicada (Perkins, 2011). En todo caso, las conclusiones obtenidas dentro de este campo contribuyen, en gran medida, al desarrollo de la teoría linguística y al de la teoría neurológica (Crystal, 1984: 31).

Gracias a los desarrollos tecnológicos actuales, "the delimitations of what is neurolinguistics has changed in terms of what parts of the brain and what parts of language and communication should be studied. Patterns and circuits of activation related to different language processing task are the main target of most neurolinguistics studies using measurement and imaging of brain activation"9 (Ahlsén, 2011: 462). Así pues, los estudios neurolingüísticos tienden a privilegiar la descripción de las zonas corticales y subcorticales y los circuitos cerebrales que intervienen durante el uso del lenguaje, ya que su objetivo central es mostrar minuciosamente la actividad cerebral durante ciertas tareas lingüísticas.

8 “[T]al comprensión es posible solo si el desorden del lenguaje es visto en relación con otros déficits centrales que puede tener el paciente."

9 "[L]as delimitaciones de lo que es neurolingüística han cambiado en términos de qué partes del cerebro y qué partes del lenguaje y la comunicación deben ser estudiadas. Los patrones y circuitos de activación relacionados con diferentes tareas de procesamiento del lenguaje son el objetivo principal de la mayoría de los estudios de neurolingüística que utilizan mediciones e imágenes de la activación cerebral." 
En resumen, la neurolingüística se ha caracterizado por el empleo de herramientas tecnológicas más sofisticadas que ayudan a ver y medir la actividad cerebral, ${ }^{10}$ así como a determinar las zonas cerebrales que intervienen durante las tareas de procesamiento linguiístico. Por otro lado, su principal fuente de estudio son las alteraciones del lenguaje causadas por daño cerebral, como las afasias (Garayzábal \& Otero, 2005; Ahlsén, 2006), y dichos resultados se emplean —en segundo término - para inferir el procesamiento del lenguaje en condiciones sin patología, lo que contribuye a los estudios psicolingüísticos. Además, en los últimos años, se han vuelto más comunes los trabajos interesados en medir la actividad cerebral en condiciones naturales, privilegiando los resultados neurofisiológicos sobre los lingüísticos. Finalmente, como resultado de los avances tecnológicos, el futuro de esta clase de investigación apunta hacia: i) una localización más precisa de las estructuras cerebrales que intervienen en el procesamiento del lenguaje, ii) la medición de la actividad cerebral en relación con el lenguaje y la comunicación ${ }^{11}$ y iii) el desarrollo filogenético del lenguaje en el cerebro, a partir - por ejemplo- del estudio de las neuronas espejo o el gen FOXP2 (Rizzolatti \& Craighero, 2004; Corballis, 2010; Ahlsén, 2011).

\section{3. ¿Cuáles son las fronteras entre psicolingüística y neurolingüística?}

Establecer límites claros es difícil, en principio porque ambas disciplinas se han nutrido una de la otra. Como ya se mencionó, la neurolingüística se ha basado en los desarrollos de la psicolingüís-

${ }^{10}$ Tomografías, resonancia magnética, electroencefalografía, potenciales evocados, magnetoencefalografía, entre otras (Ahlsén, 2006).

${ }^{11}$ A diferencia de lo que pasaba en los primeros años, ahora en neurolingüística se hace la distinción entre conducta lingüística y conducta pragmática, pues los investigadores han concluido que ambas están representadas en diferentes hemisferios cerebrales (Perkins, 2002: 2). 
tica de los últimos 30 años (Ingram, 2007) y, al mismo tiempo, los resultados provenientes del campo de la neurolingüística han contribuido a desarrollar y consolidar los postulados de la psicolingüística (Ahlsén, 2006; Field, 2011). Por tal motivo, no es de extrañar que las preguntas de investigación tiendan a traslaparse en ambas áreas (Ahlsén, 2006).

Algunos autores llegaron a considerar que ambas áreas se podían distinguir por sus objetivos y metodologías, a pesar de que "el objeto de estudio lo constituye en cualquier caso la naturaleza neuropsicológica unitaria y conjunta del lenguaje" (Fernández, 1992: 367). En este sentido, se ha subrayado que la principal diferencia es el enfoque desde el cual se aborda el objeto de estudio: la neurolingüística se centra en investigar las bases neurológicas y fisiológicas que intervienen durante la producción y comprensión del lenguaje; por ejemplo, a partir de investigaciones recientes (Kemmerer, 2015; Blake, 2017), se ha refinado la descripción del papel que desempeñan las distintas estructuras cerebrales durante el procesamiento del lenguaje, lo que ha permitido superar las explicaciones clásicas sobre la lateralización de las funciones.

En contraste, la psicolingüística presta más atención a los procesos cognitivos que participan en la adquisición, desarrollo, compresión y producción del lenguaje; es decir, está interesada en estudiar el lenguaje, pero en relación con otras funciones superiores, como atención y memoria, para determinar cómo interactúan durante el procesamiento del lenguaje. Como consecuencia, hoy sabemos que la memoria es requisito indispensable para la función del lenguaje. ${ }^{12}$

Otra forma de pensar en las diferencias entre ambas disciplinas es en términos de hardware y software, esto es, la neurolin-

${ }^{12}$ Empleando una analogía, podríamos decir que la neurolingüística haría un zoom en la estructura cerebral, mientras que realizaría un paneo en los procesos cognitivos necesarios para la función del lenguaje. En tanto que, en psicolingüística, el zoom estaría en los procesos cognitivos, mientras que el paneo incluiría las estructuras cerebrales que los alojan. 
güística trata de explicar el funcionamiento del lenguaje a partir de los elementos físicos que componen al cerebro; mientras que la psicolingüística está interesada en explicar los procesos cognitivos intangibles, pero que son indispensables para que el hardware trabaje de manera adecuada. A priori, parece que esta es una buena forma de establecer las fronteras entre ambas áreas; sin embargo, en la práctica no es tan sencillo determinar a qué área de estudio pertenece tal o cual fenómeno, por ejemplo, en el caso de la comunicación entre neuronas, ¿los impulsos eléctricos contarían como proceso o como parte de la estructura cerebral ${ }^{13}$ Así pues, a pesar de ser útil, esta distinción no está libre de polémica en cuanto a qué puede considerarse como hardware y qué como software ( $c f$. Searle, 1980; Jackendoff, 2001).

Con todo, y a pesar de los tropiezos que podamos tener al distinguir ambas áreas:

con el reconocimiento de la 'Psicolingüística' y de la 'Neurolingüística' se defiende el desarrollo de indagaciones independientes acerca del fundamento neuropsicológico de los hechos lingüísticos, partiendo de su reflejo social — propio de la Psicolingüística — y de su base material fisiológica, neuronal — propio de la Neurolingüística一, con objeto de formular hipótesis y elaborar teorías relativas a los factores, propiedades y procesos implicados en la naturaleza neuropsicológica del lenguaje (Fernández, 1992: 369-370).

Así, regresando a la pregunta inicial, parece que no hay una respuesta absoluta; en su lugar, solo podemos señalar que ambas están interesadas en las relaciones: i) cerebro-lenguaje (neurolingüística) y ii) cerebro/mente-lenguaje (psicolingüística). Del mismo modo,

${ }^{13}$ Actualmente existe un consenso sobre esto y se ha aceptado que tanto los impulsos eléctricos como las reacciones químicas en el cerebro son parte de la arquitectura cerebral y, por ende, están relacionados directamente con el hardware (Papanicolaou, 1998, 2017). 
puede afirmarse que la intervención de la lingüística, en general, se ha supeditado a la neurología y a la psicología, respectivamente, por más que se haya abogado por un estudio interdisciplinar equilibrado.

Por último, ambas disciplinas aportan significativamente a la práctica clínica, pues gracias a sus estudios experimentales se han refinado tanto los programas de rehabilitación - en el caso de las alteraciones del lenguaje-, como los criterios diagnósticos —en los casos de desarrollo típico o atípico en niños. En este contexto, la participación de la lingüística es más periférica, pues interesan conceptos como las categorías gramaticales, los tipos de oración, la estructura de la información o las operaciones morfológicas, principalmente para asociarlas con zonas de activación en el cerebro o con el fin de proponer diagramas de procesamiento, ${ }^{14}$ que tratan de emular los procesos completos de comprensión y expresión del lenguaje.

En resumen, estos estudios son de naturaleza teórica, pues los datos importan en la medida en que permiten hacer inferencias sobre cómo trabajan cerebro, mente y lenguaje, sea en condiciones típicas o atípicas. En contraste, la LC tiene un carácter más aplicado porque sus inferencias, análisis y resultados impactan directamente a la práctica clínica, ya sea refinando el diagnóstico, proponiendo un mejor programa de rehabilitación o contribuyendo con la elaboración de materiales que pueden ayudar al diagnóstico y a la rehabilitación de los pacientes. Ahondaremos sobre esto en el siguiente apartado.

\section{La lingüística clínica}

Gracias a su interés por la descripción lingüística de los síndromes afásicos, Roman Jakobson podría ser considerado como el pionero

${ }^{14}$ Dentro de la literatura psicológica y psicolingüística a este tipo de diagramas se les ha denominado boxes and arrows; las "cajas" representan un proceso cognitivo y las "flechas" indican las relaciones que se establecen entre dichos procesos. Así, la afectación de un proceso será representado por una flecha "rota". 
de los estudios en LC. Su contribución no solo incluye la elaboración de la primera tipología lingüística de las afasias, sino que, además, tuvo un enfoque verdaderamente interdisciplinar, pues siguió muy de cerca los estudios neuropsicológicos de Alexander Luria. Así, consideró que:

La aplicación de criterios puramente lingüísticos a la interpretación y clasificación de los datos sobre la afasia puede suponer una contribución esencial a la ciencia del lenguaje y de sus alteraciones, siempre que los lingüistas permanezcan tan cuidadosos y prudentes al manejar datos psicológicos y neurológicos como lo han venido siendo en su propio terreno. Ante todo, tendrían que familiarizarse con los términos y procedimientos técnicos de las disciplinas médicas que se aplican al estudio de la afasia, sometiendo los informes sobre casos clínicos a un análisis lingüístico completo, y además tendrían que trabajar ellos mismos con pacientes afásicos para conseguir una información directa, en vez de contentarse con reinterpretar observaciones concebidas y realizadas con miras muy distintas de las suyas (Jakobson, 1973 [1956]: 101-102).

No obstante, resulta curioso que solo unos cuantos autores (Powell \& Ball, 2010; Perkins \& Howard, 2011) ponderen las aportaciones de Jakobson y las consideren fundacionales para la LC. ${ }^{15}$

Tuvieron que pasar cerca de 20 años para que se volviera a abogar por la inclusión de la lingüística en el ámbito clínico con un carácter puramente aplicado, ya que su injerencia se vería reflejada — de manera directa — en el trabajo de los terapeutas. En dicho contexto resulta natural que el surgimiento de la lingüística clínica - como un área de especialidad reconocida dentro de las ciencias del lenguaje_ — es bastante reciente (Powell \& Ball, 2010).

${ }^{15}$ La introducción a Two aspects of language and two types of aphasic disturbances (Jakobson, 1956) es un verdadero tratado que sienta las bases teóricas y metodológicas para lo que después se reconocería como LC. 
Existe un consenso sobre la importancia de la obra de David Crystal en la fundación y el desarrollo de la LC contemporánea. Su trabajo durante la década de 1970 se centró en señalar la necesidad de incluir a la teoría lingüística en la práctica clínica, así como en postular los objetivos de la LC: i) describir el desarrollo normal del lenguaje, ii) describir el lenguaje en adultos sin patología, iii) describir las características lingüísticas de los desórdenes del lenguaje, iv) aportar técnicas descriptivas para ser empleadas en casos específicos de patologías de lenguaje, v) diseñar escalas evaluativas de dichas patologías, vi) proponer principios explicativos sobre adquisición y pérdida del lenguaje y vii) desarrollar obras de introducción a la lingüística dirigidas a los profesionales de otras áreas. Todas estas ideas fueron concebidas hacia 1981, cuando Crystal propuso el nombre de LC para referirse a:

the application of the theories, methods and findings of linguistics (including phonetics) to the study of those clinical situations where disorders of language are diagnosed and treated. 'Language', in this context, subsumes all four modes of interaction: speech, listening, reading and writing. 'Clinical', in this context, subsumes all those situations where there is a remedial intention involved: the initial focus is on medical settings, but educational, psychological and social settings where remedial language work is carried on are by no means excluded ${ }^{16}$ (Crystal, 1981: 1).

16 "La aplicación de las teorías, métodos y resultados de la lingüística (incluyendo la fonética) al estudio de aquellas situaciones clínicas donde se tratan y diagnostican los desórdenes del lenguaje. 'Lenguaje', en este contexto, incluye los cuatro modos de interacción: hablar, escuchar, leer y escribir. 'Clínico', en este contexto, incluye todas esas situaciones donde hay una intención terapéutica involucrada: el enfoque inicial son los entornos médicos, pero los entornos educativos, psicológicos y sociales donde se lleva a cabo el trabajo de terapia de lenguaje no están de ninguna manera excluidos." 
Veinte años después, Crystal reformuló su definición de la LC: “application of the linguistic science to the study of language disability in all its forms. The label 'disability' should not be too narrowly interpreted. It relates to anyone whose ability to use language is sufficiently undeveloped or impaired as to require special treatment or teaching" 17 (Crystal, 2001: 673); por lo que el carácter de ciencia aplicada se mantiene.

En este punto, el lector habrá advertido que la LC es necesariamente interdisciplinar, pero - a diferencia de la psicolingüística y la neurolinguiística - aquí el especialista debe estar presente en la clínica, estudiando in situ a los pacientes, pues solo así podrá decir algo real de la competencia lingüística y de la competencia comunicativa del paciente sin conformarse con descripciones parciales o sesgadas. De esta forma, podrá ser capaz de diseñar, desde una perspectiva lingüística, cuestionarios, ejercicios u otros materiales ad hoc, atendiendo a las particularidades que presente el paciente, así pues, "The scope of clinical linguistics is broad, to say the least. No level of language organisation, from phonetics to discourse, is immune to impairment, with problems manifested in both the production and comprehension of spoken, written and signed language across the human lifespan"18 (Perkins \& Howard, 2011: 111).

De tal suerte, lingüista y terapeuta trabajan — codo a codopara ofrecer un mejor servicio rehabilitatorio y/o diagnóstico (Fernández, 1998; Gallardo \& Valles, 2008; Estopà, Carrera-Sabaté \& Creus, 2010; Serra, 2013), donde, ante todo, es indispensable que

17 "[A]plicación de la ciencia lingüística al estudio de las alteraciones del lenguaje en todas sus formas. La etiqueta 'alteración' debe entenderse en un sentido amplio. Está relacionada con cualquier persona cuya capacidad para utilizar el lenguaje no esté lo suficientemente desarrollada o esté lo suficientemente deteriorada como para requerir un tratamiento o enseñanza especial."

18 "El alcance de la lingüística clínica es amplio, por decir lo menos. Ningún nivel del lenguaje, desde el fonético hasta el discursivo, es inmune al daño, que se manifiesta con problemas tanto en la producción como en la comprensión del lenguaje hablado, escrito y signado, a lo largo de la vida humana." 
exista inteligibilidad entre los profesionales de las distintas áreas; en particular, el lingüista debe tener conocimientos clínicos básicos que le permitan familiarizarse con el lenguaje técnico del terapeuta y, a su vez, ser capaz de "traducir" y explicar claramente los conceptos propios de la lingüística para que sean accesibles a los terapeutas (Pavez, 1998). Así, la "[LC] rather than a single discrete discipline it might be better seen as an interface not only between speech and language pathology and linguistics, but across a whole range of linked disciplines"19 (Perkins, 2011: 925).

En la labor clínica, el lingüista tradicionalmente se decanta por una de dos vías: "una es la lingüística clínica teórica que bebe de las evidencias empíricas, es decir, de los casos clínicos y otra es la lingüística clínica aplicada que utiliza los principios y marcos teóricos de la primera para evaluar y tratar los trastornos del lenguaje" (Marrero \& Martín, 2006: 210). En cualquier caso, los datos clínicos son fundamentales y su valor ha sido plenamente reconocido, pues sirven para evaluar teorías y metodologías lingüísticas (Howard, 2002; Müller \& Ball, 2013), pero también para generar las propias dentro del ámbito clínico y, además, tratar de explicar qué alteraciones reales se pueden presentar durante el desarrollo del lenguaje y después de una lesión cerebral (Pavez, 1998; Garayzábal, 2004). De tal forma, la LC "se preocupa directamente de aspectos relacionados con las alteraciones del lenguaje, desde un punto de vista descriptivo, identificando y dando sentido a los datos observados tanto en niños como en adultos" (Garayzábal \& Otero, 2005: 166).

Por otro lado, más recientemente, se ha postulado que el objetivo final de la LC es caracterizar las alteraciones del lenguaje de la misma forma en que la teoría lingüística general busca establecer universales linguiísticos (Crystal, 2019: 351), para lo cual es necesario realizar estudios comparativos con múltiples lenguas. Así,

19 “"[M]ás que una disciplina discreta puede ser vista como una interfaz no solo entre la patología de lenguaje y el habla y la lingüística, sino entre un rango más amplio de disciplinas relacionadas entre sí.” 
se requiere que los investigadores realicen descripciones precisas de la producción de los pacientes y que registren y clasifiquen esta información para contribuir con dicho estudio comparativo.

Crystal (2019) sostiene que la LC, como disciplina aplicada, tiene actualmente cinco metas principales: i) clarificación, relacionada con la necesidad de especificar los conceptos de las distintas disciplinas que interactúan al interior de la LC con el fin de establecer criterios y definiciones explícitos; ii) descripción, supone establecer pautas de análisis que permitan detallar la conducta lingüística de los pacientes; iii) diagnóstico, relacionado con la categorización clara de los déficits lingüísticos que presenta el paciente y que constituye una parte del proceso de diagnóstico diferencial; iv) evaluación, que implica la contribución de la LC a elaborar una evaluación más sofisticada y precisa de las alteraciones lingüísticas de los pacientes, y v) intervención, en la que, a partir del análisis de la producción y comprensión del paciente, la LC auxilia a los terapeutas clínicos a realizar una mejor intervención y a plantear programas de rehabilitación óptimos.

Así pues, la importancia de la contribución de la LC al estudio y análisis de las alteraciones del lenguaje está plenamente reconocida. No obstante, en México aún no contamos con una tradición en este tipo de estudios; más aún, en los programas de licenciatura en lingüística no se ofrecen cursos relacionados con la LC y el panorama en los posgrados tampoco es muy diferente. A continuación, profundizaremos en estas observaciones.

\section{El estudio de las alteraciones del lenguaje en México}

De acuerdo con la información de Lingmex ${ }^{20}$ (Barriga, 2018), el primer estudio interesado en el análisis de las alteraciones del len-

${ }^{20}$ Lingmex es una base de datos cuyo objetivo es reunir de forma, lo más representativa posible, la producción emanada de la investigación lingüística realizada en México por lingüistas mexicanos o extranjeros. Cabe aclarar que tomamos esta base de datos como punto de referencia, pero no como fuente única. 
guaje desde una perspectiva netamente lingüística es el de Ávila, Berruecos y Durán (1976), quienes, a partir de las ideas de Jakobson (1956) y de Luria (1964), realizaron el Cuestionario para el estudio lingüístico de las afasias (CELA), único en su género, incluso en nuestros días. Este cuestionario:

tiene como propósito general investigar las alteraciones que se presentan en la comunicación lingüística de los afásicos adultos. Este propósito tiene, a su vez, dos finalidades. En primer lugar, el cela complementa las observaciones y apoya el diagnóstico de los demás especialistas en afasiología. En segundo, el CELA, tras la evaluación de los resultados y la delimitación de las disfunciones lingüísticas del paciente, permite que la terapia del lenguaje corresponda específicamente a los problemas que se detectaron (Ávila, Berruecos \& Durán, 1976: i).

Como se puede advertir, los propósitos del CELA coinciden con los propios de la LC, por lo que podría ser considerado como un proyecto pionero en nuestro país, incluso cuando el término LC ni siquiera había sido acuñado. También es interesante notar que se habla de "alteraciones de la comunicación lingüística" y no de "alteraciones de lenguaje", con lo que se respeta la esencia de las propuestas de Jakobson y Luria.

Dos años más tarde, el propio Ávila (1977) sintetizó las ideas de Jakobson y proporcionó una explicación estructural para el análisis de los seis tipos de afasia propuestos por Luria. Como puede observarse, en estos primeros años, el interés estuvo centrado exclusivamente en las afasias, en este sentido, Raúl Ávila puede ser considerado como el iniciador de los estudios en afasiología linguiística en nuestro país, además de ser el primero en intuir el papel de la linguística aplicada al ámbito clínico.

Para la década de los 80, este tipo de estudios aumentó y se diversificó, ya no solo se estudiaba la afasia, sino que se incorporó la adquisición atípica del lenguaje y otro tipo de trastornos. Todo esto generó que la línea limítrofe entre estudios psicolingüísticos 
y neurolingüísticos se volviera difusa, por lo que podemos encontrar investigaciones que caen bajo ambos rubros ( $c f$. Lingmex). Siguiendo esta línea, nos interesa señalar solo aquellos trabajos que, a nivel teórico, contribuyeron al desarrollo de la LC.

El primero que destaca es el de Hoyos y Uriarte (1981), quienes puntualizaron la importancia de la inclusión de la teoría lingüística al campo de las patologías del lenguaje, coincidiendo con Ávila al afirmar que esto podría ayudar a caracterizar de mejor manera el tipo de alteración de lenguaje presentado por los pacientes. Y, al hacerlo, el lingüista contribuiría a ofrecer una terapia específica, acorde con los déficits particulares de cada paciente. En este mismo sentido, el trabajo de Marcos y Valdez (1986) también enfatiza el papel coadyuvante que puede desempeñar la lingüística aplicada a las alteraciones del lenguaje. Su investigación incluye ejemplos de análisis lingüístico de algunos niveles del lenguaje — como el fonológico - con lo que demuestran la utilidad de la inclusión de la teoría lingüística al campo clínico. Como podemos observar, ambos trabajos pueden considerarse como antecedentes de lo que hoy llamamos LC.

En cuanto a las investigaciones centradas en la adquisición y desarrollo atípico del lenguaje, destacan los trabajos de Esmeralda Matute (1988, 1996; Leal \& Matute, 1995), quien desde mediados de los 80 se ha interesado por el análisis de estos fenómenos desde una perspectiva neurolinguiística y neuropsicológica. Por su parte, Donna Jackson (1996; Jackson \& Maldonado, 2016; Jackson, Galeote \& Flores, 2019) ha mostrado que los resultados provenientes de la adquisición pueden ser empleados para la terapia y la rehabilitación del lenguaje y, a lo largo de los últimos años, se ha dedicado al estudio del trastorno específico del lenguaje (TEL) y al análisis lingüístico de niños con síndrome de Down. En esta misma línea, Alejandra Auza (2009; Auza, Murata, Márquez \& Morgan, 2017) ha desarrollado su investigación en torno al análisis del TEL y, más recientemente, ha abordado el estudio de niños con autismo.

Con todo, y salvo las excepciones antes mencionadas, la mayoría de las investigaciones se han centrado en llevar a cabo aná- 
lisis lingüísticos en diversos tipos de trastornos de lenguaje, pero considerando periféricamente los conceptos de la LC. En el mejor de los escenarios, el lingüista trabaja con el terapeuta - aun sin tener acceso directo a los pacientes- y se conforma con las descripciones que este último le pueda proporcionar. En el peor de los casos, el lingüista obvia el trabajo interdisciplinar y se conforma con aprender de los manuales médicos o neuropsicológicos, postura que omite que, en la práctica, es muy difícil encontrar casos ejemplares como los presentados en dichos manuales. Probablemente por esto, durante los años 90 y la primera década de los 2000, los neuropsicólogos fueron los principales interesados en investigar y analizar las alteraciones del lenguaje, ya que en la práctica clínica es muy común que asistan personas con distintas alteraciones de lenguaje. De tal suerte que profesionales de la neuropsicología y la medicina, como Alfredo Ardila, Feggy Ostrosky, Pedro Berruecos, Paz Berruecos, Luis Quintanar, Víctor Alcaraz, Yulia Solovieva, entre otros, han sido los encargados del desarrollo de la investigación de las alteraciones del lenguaje en nuestro país, aun cuando pudieran perseguir fines distintos a los de la lingüística.

Un caso aparte es el de José Marcos Ortega, quien cuenta con formación académica y profesional en ambas áreas — la médica y la lingüística- y es considerado como una figura central para el desarrollo de la neurolingüística en México (Barriga \& Parodi, 1998). Dentro del ámbito académico, Ortega ha formado tanto a lingüistas como a médicos y neuropsicólogos, quienes coinciden en señalar que sus enseñanzas han sido determinantes en la profesionalización del análisis lingüístico dentro del ámbito clínico. Así, en la práctica ha contribuido tanto al desarrollo de la neurolingüística como al de la LC, esto último sobre todo en su papel de docente (Flores-Lázaro, comunicación personal, 2018).

En la última década han surgido nuevas líneas de investigación, pero sigue siendo asignatura pendiente otorgarle carta de ciudadanía a la LC y separarla de la psicolingüística y de la neurolingüística que, como hemos visto, difieren en metodología y objetivos. Incluso ahora, los especialistas del área tenemos que decidir en 
dónde "encajar" nuestras investigaciones, dado que la categoría de "linguuística clínica" no aparece como opción temática en congresos y encuentros académicos ${ }^{21} \mathrm{o}$ en el propio Lingmex. Esto también se ve reflejado en el número reducido de estudios interesados en la aplicación in situ de la linguiística en la clínica, según Lingmex, de 1970 a la fecha solo hay 194 investigaciones centradas en patología del lenguaje y 116 en neurolingüística, esto sin considerar que pueda haber estudios que correspondan a ambos rubros. En el caso particular de las revistas mexicanas de lingüística, ${ }^{22}$ los artículos centrados en estos temas son realmente escasos, ${ }^{23}$ tal vez porque el investigador no está seguro de si hay cabida en ellas para su trabajo. Como lo señala Garayzábal:

queremos resaltar el gran número de obstáculos que debe superar la Lingüística Clínica debido al desconocimiento intradisciplinar e interdisciplinar de su campo de actuación, lo que redunda en la gran dificultad para encontrar foros comunes de interés así como lugares de publicación donde la postura y el hacer de la Lingüística Clínica se tome en serio y en sus jus-

${ }^{21}$ Por ejemplo, en el Encuentro Internacional de Lingüística en el Noroeste sí aparece en las áreas temáticas el rubro "Lingüística clínica", pero en otros, como el Congreso Nacional de la Asociación Mexicana de Lingüística Aplicada, solo aparece el rubro "Psicolingüística", a pesar de que durante el evento se puedan realizar mesas sobre lingüística clínica o neurolingüística.

${ }^{22}$ Lo mismo sucede con los libros, no se cuenta con ediciones introductorias al estudio de las alteraciones del lenguaje desde una perspectiva lingüística, como sí las hay para otras áreas de la linguíística y como también las hay en el ámbito neuropsicológico que, con todo, persigue fines distintos a los de la lingüística.

${ }^{23}$ En una búsqueda en cuatro revistas (Anuario de Letras, Nueva Revista de Filología Hispánica (NRFH), Estudios de Lingüística Aplicada (ELA) y Cuadernos de Lingüística) se encontró que cuentan, cada una, con menos de 10 artículos publicados indizados bajo los rubros: psicolingüística, neurolingüística, patología, afasia o lingüística clínica. Además, hay que considerar que varios de ellos comparten criterios de indización, por lo que suelen repetirse. 
tos términos de aportación de información relevante al mundo científico dedicado a las alteraciones del lenguaje (Garayzábal, 2004: 177).

Este panorama se explica cuando notamos que en las universidades públicas las asignaturas de neurolingüística o LC son inexistentes, incluso bajo el rubro más general de lingüística aplicada, y esta situación se mantiene en los posgrados. En estas condiciones, es posible que los neuropsicólogos sigan siendo los encargados de llevar a cabo este tipo de estudios.

Existe otro aspecto a señalar: el número de personas con alguna alteración del lenguaje va en aumento y, en la mayoría de los casos, esto representa una condicionante en la vida del paciente. Pongamos como ejemplo el caso de la afasia. En México no se cuenta con cifras exactas sobre la prevalencia e incidencia de esta enfermedad, ${ }^{24}$ tan solo sabemos que existen 401534 habitantes con alguna discapacidad para hablar o comunicarse, de los cuales el $32.8 \%$ corresponde al grupo etario entre 30 y 59 años, mientras que el 40.7\%, al grupo entre 60 y 84 años (INEGI, 2010). Cabe señalar que, gracias a los adelantos médicos y a la democratización de los tratamientos, la esperanza de vida después de sufrir un ictus o un traumatismo cráneo-encefálico es mayor que hace algunos años, por lo que un hablante con afasia tiene una mayor expectativa de vida.

Por otro lado, debido a las condiciones de vida actuales, los ictus se presentan a edades cada vez más tempranas, lo que agudiza el problema de salud pública, pues no solo hay una mayor

${ }^{24}$ En Estados Unidos, de acuerdo con la National Aphasia Association, existen más de 2000000 de personas que padecen afasia y cada año se presentan, aproximadamente, 180000 nuevos casos. En el Reino Unido, la Stroke Association estima que hay 350000 habitantes con afasia y una incidencia anual de 11400 casos nuevos, provocados por accidentes cerebrovasculares (Greener, Enderby \& Whurr, 1999). En tanto que en Alemania hay 300000 casos de hablantes con afasia, según la omics International. 
incidencia, sino que la prevalencia también va en aumento. A esto hay que agregar los efectos psicosociales de la patología: la vida del paciente cambia de forma drástica de un momento a otro, se vuelve dependiente completamente, la vida familiar se trastoca o desintegra, por lo que puede caer en una depresión aguda lo que, en casos graves, puede culminar en suicidio.

En resumen, hemos intentado justificar la importancia del desarrollo de la LC en nuestro país, principalmente señalando tres aspectos: i) durante su formación, el estudiante de linguística rara vez tiene algún curso orientado a la discusión de los objetivos, alcances y limitaciones de la LC; ii) como consecuencia, las investigaciones en LC son muy escasas, sea porque no hay investigadores interesados en ella o porque no hay revistas especializadas en nuestro país, lo que indudablemente ha frenado su difusión, y iii) en los próximos años, las alteraciones del lenguaje se convertirán en un tema de salud pública, por lo que resulta indispensable la profesionalización del análisis lingüístico en las patologías del lenguaje, tanto en el diagnóstico como en la rehabilitación y elaboración de materiales. Finalmente, cabe mencionar que el lingüista — por su formación - no está facultado para diagnosticar ni rehabilitar pacientes, para ello necesita del trabajo conjunto con el terapeuta; así pues, su labor inicial se limita a la descripción y análisis de los datos de los pacientes.

\section{Conclusiones}

Con todo lo expuesto en $\S 4$, ha quedado de manifiesto la urgencia de promover el desarrollo y la consolidación de la LC a nivel teórico en los cursos universitarios. También se ha subrayado la necesidad imperante de llevar a cabo estudios verdaderamente interdisciplinares, en los que terapeutas, neuropsicólogos y lingüistas trabajen en conjunto para ofrecer: i) diagnósticos más certeros a partir de caracterizaciones de producción lingüística de pacientes, ii) rehabilitaciones más eficaces, que atiendan los déficits específicos que presenta cada paciente e iii) instrumentos y materiales que 
puedan coadyuvar al trabajo del terapeuta y a la rehabilitación del paciente. Al proceder de esta forma, buscamos contribuir a mejorar la calidad de vida del paciente y de las personas que lo rodean, así como responder a las necesidades sociales que le corresponden a la lingüística aplicada.

La experiencia nos dice que tanto neuropsicólogos como terapeutas del lenguaje están dispuestos a colaborar con los lingüistas, a compartir sus intereses de investigación y a trabajar en conjunto para lograr una mejor rehabilitación. Incluso, es común que en las aulas y congresos de neuropsicología haya más cabida para la LC que en los propios congresos de lingüística. De tal forma, el panorama no es desalentador, solo se necesita que la LC se vaya ganando, poco a poco, un lugar dentro del quehacer lingüístico en nuestro país, lo que nos llevará a: i) proponer asignaturas en los cursos universitarios y de posgrado que incluyan a la LC, ii) contar con más investigadores interesados en el análisis de las alteraciones del lenguaje desde esta perspectiva, iii) incentivar la aparición de más investigaciones en este rubro y iv) ayudar a los clínicos a ofrecer una mejor calidad de vida a los pacientes y a sus familiares. Sirvan estas líneas para invitar a estudiantes e investigadores a contribuir con el desarrollo de la LC en México.

\section{Referencias}

Ahlsén, Elisabeth (2006). Introduction to neurolinguistics. Ámsterdam: John Benjamins.

Ahlsén, Elisabeth (2011). Neurolinguistics. En James Simpson (Ed.), The Routledge handbook of applied linguistics (pp. 460-471). Nueva York: Routledge.

Auza, Alejandra (2009). ¿Qué es el trastorno del lenguaje? Un acercamiento teórico y clínico a su definición. Lenguaje, 37(2), 365-391. doi: 10.25100/lenguaje.v37i2.4893

Auza, Alejandra; Murata, Chiharu; Márquez Caraveo, María Elena, \& Morgan, Gareth P. (2017).

Tamiz de problemas del lenguaje-TPL. Bogotá: Manual Moderno. 
Ávila Sánchez, Raúl (1977). Afasias: selección, combinación, signos y fonemas. Nueva Revista de Filología Hispánica, 26(2), 276-285. 10.24201/ nrfh.v26i2.487

Ávila Sánchez, Raúl; Berruecos Villalobos, María Paz, \& Durán, Janice (1976). Cuestionario para el estudio lingüístico de las afasias. México: El Colegio de México.

Barriga, Rebeca (Coord.) (2018). Lingmex: bibliografía lingüística de México desde 1970 (22a ed.). México: El Colegio de México. http://lingmex. colmex.mx/

Barriga, Rebeca, \& Parodi, Claudia (1998). La lingüística en México: 1980-1996.

México: El Colegio de México/ucLa.

Blake, Margaret Lehman (2017). Right-hemisphere pragmatic disorders. En Lou-

is Cummings (Ed.), Research in clinical pragmatics (pp. 243-266).

Cham: Springer.

Broca, Paul (1865). On the site of the faculty of articulated language. En Paul Eling (Ed.), Reader in the history of aphasia (pp. 56-58). Ámsterdam: John Benjamins.

Corballis, Michael C. (2010). Mirror neurons and the evolution of language. Brain and Language, 112(1), 25-35. 10.1016/j.bandl.2009.02.002

Crystal, David (1981). Clinical linguistics. Disorders of human communication.

Nueva York: Springer.

Crystal, David (1984). Linguistic encounters with language handicap. Oxford:

Blackwell.

Crystal, David (2001). Clinical linguistics. En Mark Aronoff \& Janie Ress-Miller

(Eds.), The handbook of linguistics (pp. 673-682). Oxford: Blackwell. Crystal, David (2019). Clinical linguistics. En Jack S. Damico \& Martin J. Ball (Eds.), The Sage encyclopedia of human communication sciences and disorders (pp. 351-355). Thousand Oaks: Sage.

Estopà Bagot, Rosa; Carrera-Sabaté, Josefina, \& Creus Bellet, Imma (2010). EnRaonar: Lingüística general $i$ aplicada per a la pràctica logopèdica. Barcelona: Horsori.

Fernández, Eva, \& Smith, Helen (2011). Fundamentals of psycholinguistics. Malden: Wiley-Blackwell.

Fernández Pérez, Milagros (1992). Consideraciones sobre el establecimiento y la demarcación de la neurolingüística y la psicolingüística. En Carlos 
Martín Vide (Coord.), Lenguajes naturales y lenguajes formales: Actas del VII Congreso de Lenguajes Naturales y Lenguajes Formales (pp. 367-374). Barcelona: Universidad de Barcelona.

Fernández Pérez, Milagros (1998). El papel de la teoría y de la aplicación en la construcción de disciplinas lingüísticas. El caso de la "Lingüística clínica" y áreas conexas. Revista Española de Lingüística, 28(2), 389-419.

Field, John (2004). Psycholinguistics: The key concepts. Nueva York: Routledge.

Field, John (2011). Psycholinguistics. En James Simpson (Ed.), The Routledge handbook of applied linguistics (pp. 472-486). Nueva York: Routledge.

Gallardo Paúls, Beatriz, \& Valles González, Beatriz (2008). Lingüística en contextos clínicos: la lingüística clínica. Lengua y Habla, 12(1), 32-50.

Garayzábal Heinze, Elena (2004). Las alteraciones del lenguaje desde una perspectiva lingüística: el estado de la cuestión. Revista de Logopedia, Foniatría y Audiología, 24(4) 169-177. 10.1016/S02144603(04)75800-3

Garayzábal Heinze, Elena, \& Otero Cabarcos, María Pilar (2005). Psicolingüística, neurolingüística, logopedia y lingüística clínica: juntos sí, pero no revueltos. Filología y Lingüística, 30(1), 163-185. 10.15517/rfl.v31i1.4415

Garnham, Alan; Garrod, Simon, \& Sanford, Anthony (2006). Observations on the past and future of psycholinguistics. En Matthew J. Traxler \& Morton A. Gernsbacher (Eds.), Handbook of psycholinguistics (pp. 1-18) (2 $2^{\mathrm{a}}$ ed). Ámsterdam: Academic Press.

Greener, Jenny; Enderby, Pam, \& Whurr, Renata (1999). Speech and language therapy for aphasia following stroke. Cochrane Database of Systematic Reviews, 4, 1-77. 10.1002/14651858.CD000425.

Goodglass, Harold; Kaplan, Edith, \& Barresi, Barbara (2001). The assessment of aphasia and related disorders (3a. ed). Baltimore: Lippincott, Williams and Wilkins.

Howard, Sara (2002). Clinical linguistics for students of linguistics. Centre for Languages, Linguistics and Area Studies. https://www.llas.ac.uk// resources/gpg/401 
Hoyos Arvizu, Amalia, \& Uriarte Truïllo, Margarita (1981). La lingüística en el campo de la patología del lenguaje (Tesis de licenciatura inédita). Universidad de Sonora, Hermosillo.

Ingram, John (2007). Neurolinguistics. An introduction to spoken language processing and its disorders. Cambridge: Cambridge University Press.

Instituto Nacional de Estadística y Geografía (INeGl) (2010). Datos. Salud y seguridad social. Discapacidad. https:/www.inegi.org.mx/temas/discapacidad/ Jackendoff, Ray (2001). Language in the ecology of the mind. En Paul Cobley (Ed.), The Routledge companion to semiotics and linguistics (pp. 52-65). Londres: Routledge.

Jackson, Donna (1996). Rehabilitación y terapia del lenguaje: aportes desde la adquisición del lenguaje. En Feggy Ostrosky Solís, Alfredo Ardila \& Raquel Chayo Dichi (Eds.), Rehabilitación neuropsicológica (pp. 261-286). México: Planeta.

Jackson, Donna, \& Maldonado, Ricardo (2016). El uso de conectores en niños con y sin trastorno del lenguaje. Lingüística Mexicana, 8(2), 33-55. http:// www.amla.org.mx/wp-content/uploads/2014/02/Maldonado_05.pdf Jackson, Donna; Galeote, Miguel, \& Flores Guerrero, María Fernanda (2019). The relationship between the lexicon and grammar in Spanish-speaking children with Down syndrome. En Eva Aguilar Mediavilla, Lucía Buil Legaz, Raúl López Penadés, Víctor Sanchez Azanza \& Daniel Adrover Roig (Eds.), Atypical language development in romance languages (pp. 201-217). Ámsterdam: John Benjamins.

Jakobson, Roman (1973 [1956]). Dos aspectos del lenguaje y dos tipos de trastornos afásicos. En Roman Jakobson \& Morris Hale (Eds.), Fundamentos del lenguaje (pp. 97-143) ( $2^{\mathrm{a}}$ ed). Madrid: Ayuso.

Kemmerer, David (2015). Cognitive neuroscience of language. Nueva York: Psychology Press.

Leal Herrero, Fernando, \& Matute, Esmeralda (1995). Los efectos de la edad y del grado escolar sobre la coherencia de una narración escrita por niños con problemas de aprendizaje. Salud Mental, 18(4), 10-17.

Lebrun, Yvan (1983). Issues in neurolinguistics. Language Sciences, 5(2), 241248. 10.1016/s0388-0001(83)80026-6. 
Levelt, Willem (1989). Speaking: from intention to articulation. Cambridge: MIT Press.

Luria, Alexander (1964). Factors and forms of aphasia. En A. de Reuck \& Maeve O'Connor (Eds.), Ciba Foundation Symposium on disorders of language (pp. 143-161). Londres: J. and A. Churchill.

Luria, Alexander R. (1976 [1975]). Basic problems of neurolinguistics. La Haya:

Mouton.

Marcos Ortega, José, \& Valdez Machuca, Silvia (1986). Lingüística aplicada a la descripción de los trastornos del lenguaje (Tesis de licenciatura inédita). Benemérita Universidad Autónoma de Puebla, Puebla.

Marrero Aguiar, Victoria, \& Martín Quilis, Yolanda (2006). Áreas de actuación del lingüista clínico: la formación y la práctica, dos perspectivas complementarias. En Beatriz Gallardo, Carlos Hernández \& Verónica Moreno (Coords.), Lingüística clínica y neuropsicología cognitiva. Actas del Primer Congreso Nacional de Lingüística Clínica. Vol. 2: Lingüística y evaluación del lenguaje (pp. 201-215). Valencia: Universidad de Valencia.

Matute, Esmeralda (Comp.) (1988). Neuropsicología y educación especial. Guadalajara: Unidad Editorial del Gobierno de Jalisco.

Matute, Esmeralda (1996). Un enfoque neuropsicológico para la atención de niños con problemas específicos en el aprendizaje. En Feggy Ostrosky Solís, Alfredo Ardila \& Raquel Chayo Dichi (Eds.), Rehabilitación neuropsicológica (pp. 287-317). México: Planeta.

Müller, Nicole, \& Ball, Martin J. (2013). Linguistics, phonetics, and speech-language pathology: Clinical linguistics and phonetics. En Nicole Müller \& Martin J. Ball (Eds.), Research methods in clinical linguistics and phonetics: A practical guide (pp. 1-9). Oxford: Wiley-Blackwell.

National Aphasia Association. "2016 Aphasia Awareness Survey”. https://www. aphasia.org/

omics International. Aphasia. Incidence and prevalence. https://www.omicsonline.org/germany/aphasia-peer-reviewed-pdf-ppt-articles/

Papanicolaou, Andrew C. (1998). Fundamentals of functional brain imaging: A guide to the methods and their applications on psychology and behavioral neuroscience. Lisse: Swets \& Zeitlinger. 
Papanicolaou, Andrew C. (2017). Overview of basic concepts. En Andrew C. Papanicolaou (Ed.), The Oxford handbook of functional brain imaging in neuropsychology and cognitive neurosciences (pp. 3-11). Nueva York: Oxford University Press.

Pavez, María Mercedes (1998). Lingüística aplicada a los trastornos del lenguaje. Boletín de Filología, 37(2), 953-968.

Perkins, Michael (2002). An emergent approach to clinical pragmatics. Investigations in clinical phonetics and linguistics. En Fay Windsor, Louise Kelly \& Nigel Hewlett (Eds.), Investigations in clinical phonetics and linguistics (pp. 1-14). Mahwah: Lawrence Erlbaum.

Perkins, Michael R. (2011). Clinical linguistics: Its past, present and future. Clinical Linguistics \& Phonetics, 25(11-12), 922-927. 10.3109/02699206.2011.599471

Perkins, Michael R., \& Howard, Sara (2011). Clinical linguistics. En James Simpson (Ed.), The Routledge handbook of applied linguistics (pp. 111-123). Nueva York: Routledge.

Powell, Thomas W., \& Ball, Martin J. (2010). Foreword. En Thomas W. Powell \&

Martin J. Ball (Eds.), Clinical linguistics. Critical concepts in linguistics (pp. xxix-xli). Nueva York: Routledge.

Rizzolatti, Giacomo, \& Craighero, Laila (2004). The mirror-neuron system. Annual Review of Neuroscience, 27, 169-192. 10.1146/annurev.neuro. 27.070203.144230

Runco, Mark (2001). Creativity and cognition. En Neil J. Smelser \& Paul B.

Baltes (Eds.), International encyclopedia of the social \& behavioral sciences (pp. 2892-2895). Oxford: Elsevier.

Searle, John R. (1980). Minds, brains, and programs. Behavioral and Brain Sciences, 3(3), 417-457. http://cogprints.org/7150/1/10.1.1.83.5248.pdf Serra Alegre, Enric (2013). La perspectiva funcional en lingüística clínica. LynX.

Panorámica de Estudios Lingüísticos, 12, 113-122.

Stavrakaki, Stavroula (2012). Clinical case studies in neurolinguistics and language pathology. Journal of Clinic Case Reports, 2(3), 1-2. 10.4172/2165-7920.1000e109

Stroke Association. Aphasia awareness. https://www.stroke.org.uk/what-isaphasia/aphasia-awareness 
Weismer, Gary (2006). Speech disorders. En Matthew J. Traxler \& Morton A. Gernsbacher (Eds.). Handbook of psycholinguistics (pp. 93-124) ( $2^{\mathrm{a}}$ ed.). Ámsterdam: Academic Press.

Wernicke, Carl (1874). The aphasia symptom-complex: A psychological study on an anatomical basis. En Paul Eling (Ed.), Reader in the history of aphasia (pp. 69-89). Ámsterdam: John Benjamins.

Whitaker, Harry A., \& Whitaker, Haiganoosh (Eds.) (1976). Studies in neurolinguistics.

Vol. 1. Nueva York: Academic Press. 
